\title{
Coefficients Bounds for Certain Subclass of Biunivalent Functions Associated with Ruscheweyh $q$-Differential Operator
}

\author{
Saqib Hussain, ${ }^{1}$ Shahid Khan, ${ }^{2}$ Muhammad Asad Zaighum, ${ }^{2}$ \\ Maslina Darus, ${ }^{3}$ and Zahid Shareef ${ }^{4,5}$ \\ ${ }^{1}$ Department of Mathematics, COMSATS Institute of Information Technology, Abbottabad, Pakistan \\ ${ }^{2}$ Department of Mathematics, Riphah International University, Islamabad, Pakistan \\ ${ }^{3}$ School of Mathematical Sciences, Faculty of Sciences and Technology, Universiti Kebangsaan Malaysia, \\ 43600 Bangi, Selangor, Malaysia \\ ${ }^{4}$ Foundation Program, Dhofar University, Salalah, Oman \\ ${ }^{5}$ Division of Engineering, Higher Colleges of Technology, P.O. Box 4114, Fujairah, UAE
}

Correspondence should be addressed to Saqib Hussain; saqib_math@yahoo.com

Received 16 May 2017; Accepted 27 June 2017; Published 5 September 2017

Academic Editor: Yan Xu

Copyright (C) 2017 Saqib Hussain et al. This is an open access article distributed under the Creative Commons Attribution License, which permits unrestricted use, distribution, and reproduction in any medium, provided the original work is properly cited.

We introduce in our present investigation a new subclass of analytic and biunivalent functions associated with Ruscheweyh $q$ differential operator in open unit disk $E$. We use the Faber polynomial expansions to find $n$th coefficients bounds of class of bisubordinate functions and also find initial coefficient estimates.

\section{Introduction}

Let $\mathscr{A}$ denote the class of all function $f(z)$ which is analytic in the open unit disk $E=\{z:|z|<1\}$ and has the Taylor series expansion of the following form:

$$
f(z)=z+\sum_{n=2}^{\infty} a_{n} z^{n}
$$

By $\mathcal{S}$ we mean the class of all functions in $\mathscr{A}$ which are univalent in $E$. The Koebe one-quarter theorem [1] states that the image of $E$ under every function $f$ from $\delta$ contains a disk of radius $1 / 4$. It is well known that every univalent function $f \in \mathscr{A}$ has an inverse $f^{-1}$ which is defined as

$$
\begin{aligned}
f^{-1}(f(z)) & =z, \quad z \in E, \\
f\left(f^{-1}(w)\right) & =w, \\
|w| & <r_{0}(f), \\
r_{0}(f) & \geq \frac{1}{4},
\end{aligned}
$$

where

$$
\begin{aligned}
g(w)= & f^{-1}(w) \\
= & w-a_{2} w^{2}+\left(2 a_{2}^{2}-a_{3}\right) w^{3} \\
& -\left(5 a_{2}^{3}-5 a_{2} a_{3}+a_{4}\right) w^{4}+\cdots .
\end{aligned}
$$

A function $f \in \mathscr{A}$ is said to be biunivalent in $E$ if both $f$ and $f^{-1}$ are univalent in $E$.

If the functions $f$ and $g$ are analytic in $E$, then $f$ is said to be subordinate to $g$, written as

$$
f(z) \prec g(z), \quad z \in E
$$

if there exists a Schwarz function $w(z)$, analytic in $E$, with $w(0)=0$ and $|w(z)|<1$, such that

$$
f(z)=g(w(z)), \quad z \in E .
$$

Let $\Sigma$ denote the class of analytic and biunivalent functions in $E$ given by the Taylor-Maclaurin series expansion (1). 
Here we give few examples of functions in the class $\Sigma$ such that

$$
\begin{aligned}
& u_{1}(z)=\frac{z}{1-z}, \\
& u_{2}(z)=-\log (1-z), \\
& u_{3}(z)=\frac{1}{2} \log \left(\frac{1+z}{1-z}\right),
\end{aligned}
$$

$z \in E$.

However, the famous Koebe function $k(z)=z /(1-z)^{2}$ is not in $\Sigma$; for more details we refer to [2]. For $f \in \Sigma$, the class of biunivalent analytic functions was first introduced and studied by Lewin where it was proved to show that $\left|a_{2}\right|<1.51$. Brannan and Clunie [3] proved that $\left|a_{2}\right| \leq \sqrt{2}$. Netanyahu [4] showed that $\max \left|a_{2}\right|=4 / 3$. Brannan and Taha [5] introduced certain subclass of the biunivalent functions class $\Sigma$. For a brief history and interesting examples of biunivalent functions we refer to [3, 5-11].

The Faber polynomials introduced by Faber [12]. Gong [13] and Schiffer [14] demonstrated the significance of the Faber polynomials in mathematical sciences, especially in geometric function theory. In the literature, there are only a few works determining the general coefficient bounds $\left|a_{n}\right|$ for the analytic biunivalent functions given by (1) using Faber polynomial expansions. A very little is known about the bounds of Maclaurin's series coefficient $\left|a_{n}\right|$ for $n \geq 3$ by using a Faber polynomials. For more study about Faber polynomials we refer to [15-21].

Using the technique of convolution, Ruscheweyh [22] defined the operator $R^{\lambda}$ on the class of analytic functions $\mathscr{A}$ as

$$
R^{\lambda} f(z)=f(z) * \frac{z}{(1-z)^{\lambda+1}}
$$

$$
z \in E, \lambda \in \mathbb{R}, \lambda>-1
$$

For $\lambda=m \in N_{0}=N U\{0\}$, we obtain

$$
R^{m} f(z)=\frac{z\left(z^{m-1} f(z)\right)^{(m)}}{m !} .
$$

The expression $R^{m} f(z)$ is called an $m$ th-order Ruscheweyh derivative of $f(z)$ and the symbol $*$ stands for Hadamard product (or convolution).

For $t \in \mathbb{R}$ and $q>0, q \neq 1$, the number $[t]$ is defined in $[23]$ as

$$
[t]=\frac{1-q^{t}}{1-q}, \quad[0]=0 .
$$

For any nonnegative integer $n$ the $q$-number shift factorial is defined as

$$
[n] !=[1][2][3] \cdots[n], \quad([0] !=1) .
$$

We have $\lim _{q \rightarrow 1}[n]=n$. Throughout in this paper we will assume $q$ to be fixed number between 0 and 1 .
The $q$-derivative operator or $q$-difference operator for $f \in$ $A$ is defined as

$$
\partial_{q} f(z)=\frac{f(q z)-f(z)}{z(q-1)}, \quad z \in E .
$$

It can easily be seen that for $n \in N=\{1,2,3, \ldots\}$ and $z \in E$.

$$
\begin{aligned}
\partial_{q} z^{n} & =[n] z^{n-1}, \\
\partial_{q}\left\{\sum_{n=1}^{\infty} a_{n} z^{n}\right\} & =\sum_{n=1}^{\infty}[n] a_{n} z^{n-1}
\end{aligned}
$$

The $q$-generalized Pochhammer symbol for $t \in R$ and $n \in N$ is defined as

$$
[t]_{n}=[t][t+1][t+2] \cdots[t+(n-1)],
$$

and, for $t>0$, let $q$-gamma function be defined as

$$
\begin{aligned}
\Gamma_{q}(t+1) & =[t] \Gamma_{q}(t), \\
\Gamma_{q}(1) & =1 .
\end{aligned}
$$

For $f \in \mathscr{A}$ Ruscheweyh $q$-differential operator was defined by Aldweby and Darus [24] (see also [23]), as

$$
\begin{aligned}
R_{q}^{\lambda} f(z) & =f(z) * F_{q, \lambda+1}(z), \quad z \in E, \lambda>-1 . \\
F_{q, \lambda+1}(z) & =z+\sum_{n=2}^{\infty} \frac{\Gamma_{q}(n+\lambda)}{[n-1] ! \Gamma_{q}(1+\lambda)} z^{n} \\
& =z+\sum_{n=2}^{\infty} \frac{[\lambda+1]_{n-1}}{[n-1] !} z^{n} \\
& =z+\sum_{n=2}^{\infty} \Psi_{n-1} z^{n}, \\
\Psi_{n-1} & =\frac{\Gamma_{q}(n+\lambda)}{[n-1] ! \Gamma_{q}(1+\lambda)}=\frac{[\lambda+1]_{n-1}}{[n-1] !} \\
z \partial_{q}\left(R_{q}^{\lambda} f(z)\right) & =\left(1+\frac{[\lambda]}{q^{\lambda}}\right) R_{q}^{\lambda+1} f(z)-\frac{[\lambda]}{q^{\lambda}} R_{q}^{\lambda} f(z) .
\end{aligned}
$$

If $q \rightarrow 1$, equality (17) implies

$$
z\left(R^{\lambda} f(z)\right)^{\prime}=(1+\lambda) R^{\lambda+1} f(z)-\lambda R^{\lambda} f(z) .
$$

which is the well known recurrent formula for Ruscheweyh differential operator.

in the present paper we introduce new subclass of the function class $\Sigma$, involving Ruscheweyh $q$-differential operator $R_{q}^{\lambda} f(z)$. By using Faber polynomial coefficient techniques we determine estimates for the general coefficient bounds $\left|a_{n}\right|$ for $n \geq 3$ and also estimates on the coefficients $\left|a_{2}\right|$ and $\left|a_{3}\right|$ for functions in the new subclass of function class $\sum$. Several related classes are also considered, and connections to earlier known results are also defined. 
Definition 1. A function $f \in \Sigma, 0 \leq \gamma,-1<\lambda, 1 \leq \beta$, and $0 \leq \alpha<1$; we introduce a new class of biunivalent functions $\mathcal{N}_{\Sigma}^{q(\boldsymbol{\alpha}, \boldsymbol{\beta}, \boldsymbol{\gamma}, \lambda)}$ as $f \in \mathcal{N}_{\Sigma}^{q(\boldsymbol{\alpha}, \boldsymbol{\beta}, \boldsymbol{\gamma}, \lambda)}$ if and only if

$$
\begin{aligned}
& \frac{(1-\beta) R_{q}^{\lambda} f(z)+\beta z \partial_{q} R_{q}^{\lambda} f(z)+\gamma z^{2} \partial_{q}^{2} R_{q}^{\lambda} f(z)}{z} \\
& <\frac{1+A z}{1+B z}, \quad z \in E, \\
& \frac{(1-\beta) R_{q}^{\lambda} g(w)+\beta w \partial_{q} R_{q}^{\lambda} g(w)+\gamma w^{2} \partial_{q}^{2} R_{q}^{\lambda} g(w)}{w} \\
& \prec \frac{1+A w}{1+B w}, \quad w \in E,
\end{aligned}
$$

where

$$
\begin{aligned}
& A=\{1-\alpha(1+q)\}, \\
& B=-q
\end{aligned}
$$

and $g(w)=f^{-1}(w)$ is defined by (3).

On specializing the parameters $\alpha, \beta$, and $\gamma$, one can state the various new subclasses as illustrated in the following definition.

Definition 2. For $\beta=1,-1<\lambda, 0 \leq \gamma$, and $0 \leq \alpha<1$. A function, $f \in \Sigma$, is in the class $\mathcal{N}_{\Sigma}^{q(\alpha, \gamma, \lambda)}$ if the following conditions are satisfied:

$$
\begin{gathered}
\partial_{q} R_{q}^{\lambda} f(z)+\gamma z \partial_{q}^{2} R_{q}^{\lambda} f(z) \prec \frac{1+A z}{1+B z}, \quad z \in E, \\
\partial_{q} R_{q}^{\lambda} g(w)+\gamma w \partial_{q}^{2} R_{q}^{\lambda} g(w) \prec \frac{1+A w}{1+B w}, \quad w \in E,
\end{gathered}
$$

where $A, B$ are given by (20), and $g(w)=f^{-1}(w)$ is defined by (3).

Definition 3. For $\beta=1, \gamma=0,-1<\lambda$, and $0 \leq \alpha<1$. A function, $f \in \Sigma$, is in the class $\mathcal{N}_{\Sigma}^{q(\boldsymbol{\alpha}, \lambda)}$ if the following conditions are satisfied:

$$
\begin{aligned}
& \partial_{q} R_{q}^{\lambda} f(z) \prec \frac{1+A z}{1+B z}, \quad z \in E, \\
& \partial_{q} R_{q}^{\lambda} g(w) \prec \frac{1+A w}{1+B w}, \quad w \in E,
\end{aligned}
$$

where $A, B$ are given by $(20)$ and $g(w)=f^{-1}(w)$ is defined by (3).

Definition 4. For $\gamma=0,-1<\lambda$ and $0 \leq \alpha<1$. A function, $f \in \Sigma$, is in the class $\mathscr{N}_{\Sigma}^{q(\boldsymbol{\alpha}, \boldsymbol{\beta}, \lambda)}$ if the following conditions are satisfied:

$$
\begin{aligned}
& (1-\beta) \frac{R_{q}^{\lambda} f(z)}{z}+\beta \partial_{q} R_{q}^{\lambda} f(z) \prec \frac{1+A z}{1+B z}, \quad z \in E, \\
& (1-\beta) \frac{R_{q}^{\lambda} g(w)}{w}+\beta \partial_{q} R_{q}^{\lambda} g(w) \prec \frac{1+A w}{1+B w}, \quad w \in E,
\end{aligned}
$$

where $A, B$ are given by (20) and $g(w)=f^{-1}(w)$ is defined by (3).
It is well known that

$$
f(z) \prec \frac{1+(1-2 \alpha) z}{1-z} \quad \text { iff } \operatorname{Re}(f(z))>\alpha .
$$

Special Cases

(i) For $\lambda=0$ and $q \rightarrow 1$, the class $\mathscr{N}_{\Sigma}^{q(\boldsymbol{\alpha}, \boldsymbol{\beta}, \gamma, \lambda)}=\mathcal{N}_{\Sigma}^{(\boldsymbol{\alpha}, \boldsymbol{\beta}, \boldsymbol{\gamma})}$; see $[21]$.

(ii) For $\beta=1, \lambda=0$, and $q \rightarrow 1$, the class $\mathcal{N}_{\Sigma}^{q(\boldsymbol{\alpha}, \boldsymbol{\beta}, \boldsymbol{\gamma}, \boldsymbol{\lambda})}=$ $\mathscr{B}_{\Sigma}^{(\boldsymbol{\alpha}, \boldsymbol{\gamma})}$; see [25].

(iii) For $\beta=1, \lambda=0, \gamma=0$, and $q \rightarrow 1$, the class $\mathscr{N}_{\Sigma}^{q(\boldsymbol{\alpha}, \boldsymbol{\beta}, \boldsymbol{\gamma}, \lambda)}=\mathscr{H}_{\Sigma}^{\boldsymbol{\alpha}} ;$ see [2].

(iv) For $\lambda=0, \gamma=0$, and $q \rightarrow 1$, the class $\mathscr{N}_{\Sigma}^{q(\boldsymbol{\alpha}, \boldsymbol{\beta}, \boldsymbol{\gamma}, \boldsymbol{\lambda})}=$ $\mathcal{N}_{\Sigma}^{(\boldsymbol{\alpha}, \boldsymbol{\beta})}$; see [26].

Lemma 5 (see [27]). Let the Schwarz function $w(z)$ be given by

$$
w(z)=w_{1} z+w_{2} z^{2}+w_{3} z^{3}+\cdots, \quad(z \in E)
$$

then

$$
\begin{gathered}
\left|w_{1}\right| \leq 1 \\
\left|w_{2}\right| \leq 1-\left|w_{1}\right|^{2}, \\
\left|w_{2}-t w_{1}^{2}\right| \leq 1+(|t|-1)\left|w_{1}\right|^{2} .
\end{gathered}
$$

\section{Main Results}

Using the Faber polynomial expansion of functions $f \in A$ of the form (1), the coefficients of its inverse map $g=f^{-1}$ may be expressed as [16] given by

$$
g(w)=f^{-1}(w)=w+\sum_{n=2}^{\infty} \frac{1}{n} K_{n-1}^{-n}\left(a_{2}, a_{3}, \ldots\right) w^{n}
$$

where

$$
\begin{aligned}
K_{n-1}^{-n} & \\
= & \frac{(-n) !}{(-2 n+1) !(n-5) !} a_{2}^{n-1} \\
& +\frac{(-n) !}{[2(-n+1)] !(n-3) !} a_{2}^{n-3} a_{3} \\
& +\frac{(-n) !}{(-2 n+3) !(n-4) !} a_{2}^{n-4} a_{4} \\
& +\frac{(-n) !}{[2(-n+2)] !(n-5) !} a_{2}^{n-5}\left[a_{5}+(-n+2) a_{3}^{2}\right] \\
& +\frac{(-n) !}{(-2 n+5) !(n-6) !} a_{2}^{n-6}\left[a_{6}+(-2 n+5) a_{3} a_{4}\right] \\
& +\sum_{j \geq 7} a_{2}^{n-j} V_{j},
\end{aligned}
$$


such that $V_{j}$ with $7 \leq j \leq n$ is a homogeneous polynomial in the variables $\left|a_{2}\right|,\left|a_{3}\right|, \ldots,\left|a_{n}\right|$; see [28]. In particular, the first three terms of $K_{n-1}^{-n}$ are in general, for any $p \in N$ and $n \geq 2$, an expansion of $K_{n-1}^{p}$ (for details we refer [16, 29]), which is as follows:

$$
\begin{aligned}
K_{n-1}^{p}= & p a_{n}+\frac{p(p-1)}{2} E_{n-1}^{2}+\frac{p !}{(p-3) ! 3 !} E_{n-1}^{3}+\cdots \\
& +\frac{p !}{(p-n+1) !(n-1) !} E_{n-1}^{n-1},
\end{aligned}
$$

where $E_{n-1}^{p}=E_{n-1}^{p}\left(a_{2}, a_{3}, \ldots\right)$ and, by [28],

$$
E_{n-1}^{m}\left(a_{2}, \ldots, a_{n}\right)=\sum_{n=2}^{\infty} \frac{m !\left(a_{2}\right)^{\mu_{1}} \cdots\left(a_{n}\right)^{\mu_{n-1}}}{\mu_{1 !}, \ldots, \mu_{n-1} !}
$$

for $m \leq n$,

while $a_{1}=1$, and the sum is taken over all nonnegative integer $\mu_{1}, \ldots, \mu_{n}$ satisfying

$$
\begin{aligned}
\mu_{1}+\mu_{2}+\cdots+\mu_{n} & =m, \\
\mu_{1}+2 \mu_{2}+\cdots+(n-1) \mu_{n-1} & =n-1 .
\end{aligned}
$$

Evidently, $E_{n-1}^{n-1}\left(a_{2}, \ldots, a_{n}\right)=a_{2}^{n-1}$ [15], or, equivalently,

$$
E_{n}^{m}\left(a_{1}, a_{2}, \ldots, a_{n}\right)=\sum_{n=1}^{\infty} \frac{m !\left(a_{1}\right)^{\mu_{1}} \cdots\left(a_{n}\right)^{\mu_{n}}}{\mu_{1 !}, \ldots, \mu_{n} !},
$$

for $m \leq n$,

while $a_{1}=1$, and the sum is taken over all nonnegative integer $\mu_{1}, \ldots, \mu_{n}$ satisfying

$$
\begin{aligned}
\mu_{1}+\mu_{2}+\cdots+\mu_{n} & =m, \\
\mu_{1}+2 \mu_{2}+\cdots+(n) \mu_{n} & =n .
\end{aligned}
$$

It is clear that $E_{n}^{n}\left(a_{1}, \ldots, a_{n}\right)=E_{1}^{n}$; the first and last polynomials are $E_{n}^{n}=a_{1}^{n}, E_{n}^{1}=a_{n}$.

Theorem 6. For $0 \leq \gamma, 1 \leq \beta,-1<\lambda$, and $0 \leq \alpha<1$. If $f \in \mathscr{N}_{\Sigma}^{q(\boldsymbol{\alpha}, \boldsymbol{\beta}, \gamma, \lambda)}$, if $a_{m}=0,2 \leq m \leq n-1$, then

$$
\left|a_{n}\right| \leq \frac{(1-\alpha)(1+q)[n-1] ! \Gamma_{q}(1+\lambda)}{\{(1-\beta)+\beta[n]+\gamma[n][n-1]\} \Gamma_{q}(n+\lambda)},
$$

for $n \geq 3$.

Proof. For the function $f \in \mathcal{N}_{\Sigma}^{q(\boldsymbol{\alpha}, \boldsymbol{\beta}, \boldsymbol{\gamma}, \boldsymbol{\lambda})}$ of the form (1), we have

$$
\begin{aligned}
& \frac{(1-\beta) R_{q}^{\lambda} f(z)+\beta\left(z \partial_{q} R_{q}^{\lambda} f(z)+\gamma z^{2} \partial_{q}^{2} R_{q}^{\lambda} f(z)\right)}{z} \\
& =1+\sum_{n=2}^{\infty}\{(1-\beta)+\beta[n]+\gamma[n][n-1]\} \\
& \quad \times \Psi_{n-1} a_{n} z^{n-1},
\end{aligned}
$$

and, for its inverse map $g=f^{-1}$, we have

$$
\begin{aligned}
& \frac{(1-\beta) R_{q}^{\lambda} g(w)+\beta\left(w \partial_{q} R_{q}^{\lambda} g(w)+\gamma w^{2} \partial_{q}^{2} R_{q}^{\lambda} g(w)\right)}{w} \\
& =1+\sum_{n=2}^{\infty}\{(1-\beta)+\beta[n]+\gamma[n][n-1]\} \\
& \quad \times \Psi_{n-1} b_{n} w^{n-1},
\end{aligned}
$$

where $\Psi_{n-1}$ is given by $(16)$ and $b_{n}=(1 / n) K_{n-1}^{-n}\left(a_{2}, a_{3}, \ldots, a_{n}\right)$.

Since both function $f$ and its inverse map $g=f^{-1}$ are in $\mathcal{N}_{\Sigma}^{q(\boldsymbol{\alpha}, \boldsymbol{\beta}, \boldsymbol{\gamma}, \boldsymbol{\lambda})}$, by the definition of subordination there exist two Schwarz functions $p(z)=\sum_{n=1}^{\infty} c_{n} z^{n}$ and $q(w)=\sum_{n=1}^{\infty} d_{n} w^{n}$, where $z, w \in E$. We have

$$
\begin{aligned}
& \frac{(1-\beta) R_{q}^{\lambda} f(z)+\beta\left(z \partial_{q} R_{q}^{\lambda} f(z)+\gamma z^{2} \partial_{q}^{2} R_{q}^{\lambda} f(z)\right)}{z} \\
& \quad=\frac{1+A(p(z))}{1+B(p(z))},
\end{aligned}
$$

where

$$
\begin{aligned}
\frac{1+A(p(z))}{1+B(p(z))} & \\
& =1-\sum_{n=1}^{\infty}(A-B) K_{n}^{-1}\left(c_{1}, c_{2}, \ldots, c_{n}, B\right) z^{n}, \\
\frac{(1-\beta) R_{q}^{\lambda} g(w)+\beta\left\{w \partial_{q} R_{q}^{\lambda} g(w)+\gamma w^{2} \partial_{q}^{2} R_{q}^{\lambda} g(w)\right\}}{w} & \\
= & \frac{1+A(q(w))}{1+B(q(w))},
\end{aligned}
$$

where

$$
\begin{aligned}
\frac{1+A(q(w))}{1+B(q(w))} \\
\quad=1-\sum_{n=1}^{\infty}(A-B) K_{n}^{-1}\left(d_{1}, d_{2}, \ldots, d_{n}, B\right) w^{n} .
\end{aligned}
$$

In general $[15,16]$ for any $p \in N$ and $n \geq 2$, an expansion of $K_{n}^{p}\left(k_{1}, k_{2}, \ldots, k_{n}, B\right)$,

$$
\begin{gathered}
K_{n}^{p}\left(k_{1}, k_{2}, \ldots, k_{n}, B\right)=\frac{p !}{(p-n) ! n !} k_{1}^{n}(B)^{n-1} \\
+\frac{p !}{(p-n+1) !(n-2) !} k_{1}^{n-2} k_{2}(B)^{n-2} \\
+\frac{p !}{(p-n+2) !(n-3) !} \times k_{1}^{n-3} k_{3}(B)^{n-3}
\end{gathered}
$$




$$
\begin{aligned}
& +\frac{p !}{(p-n+3) !(n-4) !} \\
& \cdot k_{1}^{n-4}\left[k_{4}(B)^{n-4}+\frac{p-n+3}{2} k_{3}^{2}(B)\right] \\
& +\frac{p !}{(p-n+4) !(n-5) !} \\
& \cdot k_{1}^{n-5}\left[k_{5}(B)^{n-5}+(p-n+4) k_{3} k_{4}(B)\right] \\
& +\sum_{j \geq 6} k_{1}^{n-1} X_{j},
\end{aligned}
$$

where $X_{j}$ is a homogeneous polynomial of degree $j$ in the variables $k_{1}, k_{2}, \ldots, k_{n}$.

For the coefficients of the Schwarz functions $p(z)$ and $q(w),\left|c_{n}\right| \leq 1$ and $\left|d_{n}\right| \leq 1$ [9].

Comparing the corresponding coefficients of (35) and (37), we have

$$
\begin{aligned}
& \{(1-\beta)+\beta[n]+\gamma[n][n-1]\} \Psi_{n-1} a_{n} \\
& \quad=-(A-B) K_{n-1}^{-1}\left(c_{1}, c_{2}, \ldots, c_{n-1}, B\right) .
\end{aligned}
$$

Similarly, corresponding to coefficients of (36) and (39), we have

$$
\begin{aligned}
& \{(1-\beta)+\beta[n]+\gamma[n][n-1]\} \Psi_{n-1} b_{n} \\
& \quad=-(A-B) K_{n-1}^{-1}\left(d_{1}, d_{2}, \ldots, d_{n-1}, B\right) .
\end{aligned}
$$

Note that, for $a_{m}=0,2 \leq m \leq n-1$, we have $b_{n}=-a_{n}$

$$
\begin{aligned}
& \{(1-\beta)+\beta[n]+\beta \gamma[n][n-1]\} \Psi_{n-1} a_{n} \\
& \quad=-(A-B) c_{n-1}, \\
& -\{(1-\beta)+\beta[n]+\beta \gamma[n][n-1]\} \Psi_{n-1} a_{n} \\
& \quad=-(A-B) d_{n-1} .
\end{aligned}
$$

Taking the absolute values of (44) and (45), we have

$$
\begin{aligned}
\left|a_{n}\right| & \leq \frac{|A-B|}{\{(1-\beta)+\beta[n]+\gamma[n][n-1]\} \Psi_{n-1}}\left|c_{n-1}\right| \\
& =\frac{|A-B|}{\{(1-\beta)+\beta[n]+\gamma[n][n-1]\} \Psi_{n-1}}\left|d_{n-1}\right|, \\
\left|a_{n}\right| & \leq \frac{|A-B|}{\{(1-\beta)+\beta[n]+\gamma[n][n-1]\} \Psi_{n-1}},
\end{aligned}
$$

for $n \geq 3$.
By using $A=\{1-\alpha(1+q)\}, B=-q$, from (20) and $\Psi_{n-1}=$ $\Gamma_{q}(n+\lambda) /[n-1] ! \Gamma_{q}(1+\lambda)$ from (16) in (47), we have

$$
\left|a_{n}\right| \leq \frac{(1-\alpha)(1+q)[n-1] ! \Gamma_{q}(1+\lambda)}{\{(1-\beta)+\beta[n]+\gamma[n][n-1]\} \Gamma_{q}(n+\lambda)},
$$

for $n \geq 3$,

which completes the proof of theorem.

By putting $\beta=1$, in Theorem 6 , we have the following corollary.

Corollary 7. For $0 \leq \gamma,-1<\lambda$, and $0 \leq \alpha<1$, if $f \in$ $\mathcal{N}_{\Sigma}^{q(\boldsymbol{\alpha}, \gamma, \lambda)}$ and if $a_{m}=0,2 \leq m \leq n-1$, then

$$
\left|a_{n}\right| \leq \frac{(1-\alpha)(1+q)[n-1] ! \Gamma_{q}(1+\lambda)}{[n]\{1+\gamma[n-1]\} \Gamma_{q}(n+\lambda)}, \text { for } n \geq 3 \text {. }
$$

For $\beta=1, \gamma=0$, in Theorem 6 , we have the following corollary.

Corollary 8. For $0 \leq \alpha<1,-1<\lambda$, if $f \in \mathcal{N}_{\Sigma}^{q(\boldsymbol{\alpha}, \lambda)}$ and if $a_{m}=0,2 \leq m \leq n-1$, then

$$
\left|a_{n}\right| \leq \frac{(1-\alpha)(1+q)[n-1] ! \Gamma_{q}(1+\lambda)}{[n] \Gamma_{q}(n+\lambda)}, \text { for } n \geq 3 .
$$

For $\lambda=0, q \rightarrow 1$ in Theorem 6, we obtain the following corollary.

Corollary 9 (see [21]). For $1 \leq \beta$ and $0 \leq \gamma, 0 \leq \alpha<1$, if $f \in \mathcal{N}_{\Sigma}^{(\boldsymbol{\alpha}, \boldsymbol{\beta}, \boldsymbol{\gamma})}$ and if $a_{m}=0,2 \leq m \leq n-1$, then

$$
\left|a_{n}\right| \leq \frac{2(1-\alpha)}{1+\beta(n-1)+\gamma n(n-1)}, \text { for } n \geq 3 .
$$

For $\beta=1, \lambda=0$, and $q \rightarrow 1$ in Theorem 6 , we obtain the following corollary.

Corollary 10 (see [25]). For $0 \leq \alpha<1$ and $0 \leq \gamma$, if $f \in$ $\mathscr{B}_{\Sigma}^{(\boldsymbol{\alpha}, \boldsymbol{\gamma})}$ and if $a_{m}=0,2 \leq m \leq n-1$, then

$$
\left|a_{n}\right| \leq \frac{2(1-\alpha)}{n\{1+\gamma(n-1)\}}, \quad \text { for } n \geq 3 .
$$

For $\beta=1, \gamma=0, \lambda=0, q \rightarrow 1$ in Theorem 6 , we obtain the following corollary.

Corollary 11. For $0 \leq \alpha<1$, if $f \in \mathscr{H}_{\Sigma}^{(\boldsymbol{\alpha})}$ and if $a_{m}=0$, $2 \leq m \leq n-1$, then

$$
\left|a_{n}\right| \leq \frac{2(1-\alpha)}{n}, \text { for } n \geq 3 .
$$

For $\gamma=0, \lambda=0, q \rightarrow 1$ in Theorem 6 , we obtain the following corollary.

Corollary 12 (see [30]). For $1 \leq \beta$ and $0 \leq \alpha<1$, if $f \in$ $\mathcal{N}_{\Sigma}^{(\boldsymbol{\alpha}, \boldsymbol{\beta})}$ and if $a_{m}=0,2 \leq m \leq n-1$, then

$$
\left|a_{n}\right| \leq \frac{2(1-\alpha)}{1+(n-1) \beta}, \text { for } n \geq 3 \text {. }
$$

Theorem 13. For $0 \leq \alpha<1,-1<\lambda, 1 \leq \beta$, and $0 \leq \gamma$, if $f \in \mathcal{N}_{\Sigma}^{q(\boldsymbol{\alpha}, \boldsymbol{\beta}, \boldsymbol{\gamma}, \boldsymbol{\lambda})}$, then 


$$
\begin{aligned}
& \left|a_{2}\right| \leq \min \left\{\begin{array}{l}
\frac{(1-\alpha)(1+q)[1] ! \Gamma_{q}(1+\lambda)}{\{(1-\beta)+\beta[2]+\gamma[2][1]\} \Gamma_{q}(2+\lambda)} \\
\sqrt{\frac{2|q(1-\alpha)(1+q)|[2] ! \Gamma_{q}(1+\lambda)}{[2]\{(1-\beta)+\beta[3]+\gamma[3][2]\} \Gamma_{q}(3+\lambda)}}
\end{array}\right. \\
& \left|a_{3}\right| \leq \min \left\{\begin{array}{l}
\frac{[2]}{([1]+[1])}\left[\frac{(1-\alpha)(1+q)[1] ! \Gamma_{q}(1+\lambda)}{\{(1-\beta)+\beta[2]+\gamma[2][1]\} \Gamma_{q}(2+\lambda)}\right]^{2}, \\
\frac{[2]}{([1]+[1])}\left[\frac{2 q(1-\alpha)(1+q)[2] ! \Gamma_{q}(1+\lambda)}{[2]\{(1-\beta)+\beta[3]+\gamma[3][2]\} \Gamma_{q}(3+\lambda)}\right],
\end{array}\right. \\
& \left|a_{3}-[2] a_{2}^{2}\right| \leq \frac{q(1-\alpha)(1+q)[2] ! \Gamma_{q}(1+\lambda)}{\{(1-\beta)+\beta[3]+\gamma[3][2]\} \Gamma_{q}(3+\lambda)}
\end{aligned}
$$

Proof. Replacing $n$ by 2 and 3 in (44) and (45), respectively, we have

$$
\begin{aligned}
& \{(1-\beta)+\beta[2]+\gamma[2][1]\} \Psi_{1} a_{2}=-(A-B) c_{1}, \\
& \{(1-\beta)+\beta[3]+\gamma[3][2]\} \Psi_{2} a_{3} \\
& \quad=-(A-B)\left(B c_{1}^{2}-c_{2}\right), \\
& -\{(1-\beta)+\beta[2]+\gamma[2][1]\} \Psi_{1} a_{2}=-(A-B) d_{1}, \\
& \{(1-\beta)+\beta[3]+\gamma[3][2]\} \Psi_{2}\left\{[2] a_{2}^{2}-a_{3}\right\} \\
& \quad=-(A-B)\left(B d_{1}^{2}-d_{2}\right) .
\end{aligned}
$$

From (56) and (58) we have

$$
\begin{aligned}
c_{1} & =-d_{1}, \\
\left|a_{2}\right| & \leq \frac{|A-B|}{\{(1-\beta)+\beta[2]+\gamma[2][1]\} \Psi_{1}}\left|c_{1}\right| \\
& =\frac{|A-B|}{\{(1-\beta)+\beta[2]+\gamma[2][1]\} \Psi_{1}}\left|d_{1}\right|, \\
& \leq \frac{|A-B|}{\{(1-\beta)+\beta[2]+\gamma[2][1]\} \Psi_{1}} .
\end{aligned}
$$

Using (20) and (16) in (61), we have

$$
\left|a_{2}\right| \leq \frac{(1-\alpha)(1+q)[1] ! \Gamma_{q}(1+\lambda)}{\{(1-\beta)+\beta[2]+\gamma[2][1]\} \Gamma_{q}(2+\lambda)} .
$$

Adding (57) and (59) we have

$$
\begin{aligned}
{[2] } & \{(1-\beta)+\beta[3]+\gamma[3][2]\} \Psi_{2} a_{2}^{2} \\
& =-(A-B)\left\{B\left(c_{1}^{2}+d_{1}^{2}\right)-\left(c_{2}+d_{2}\right)\right\} .
\end{aligned}
$$

Taking absolute values of both sides of (63) and applying the estimates $\left|c_{2}\right| \leq 1-\left|c_{1}\right|^{2},\left|d_{2}\right| \leq 1-\left|d_{1}\right|^{2}$ of Lemma 5 and $\left|c_{1}\right| \leq 1,\left|d_{1}\right| \leq 1$, we have

$$
\begin{aligned}
& \left|a_{2}\right|^{2} \leq \frac{2|A-B||B|}{[2]\{(1-\beta)+\beta[3]+\gamma[3][2]\} \Psi_{2}}, \\
& \left|a_{2}\right| \leq \sqrt{\frac{2|A-B||B|}{[2]\{(1-\beta)+\beta[3]+\gamma[3][2]\} \Psi_{2}}} ;
\end{aligned}
$$

using (20) and (16) in (64), we have

$$
\left|a_{2}\right| \leq \sqrt{\frac{2 q(1-\alpha)(1+q)[2] ! \Gamma_{q}(1+\lambda)}{[2]\{(1-\beta)+\beta[3]+\gamma[3][2]\} \Gamma_{q}(3+\lambda)}} .
$$

Now, in order to find $\left|a_{3}\right|$, we subtract (59) from (57) and we have

$$
\begin{aligned}
a_{3}= & \frac{(A-B)\left\{B\left(d_{1}^{2}-c_{1}^{2}\right)-\left(c_{2}-d_{2}\right)\right\}}{([1]+[1])\{(1-\beta)+\beta[3]+\gamma[3][2]\} \Psi_{2}} \\
& +\frac{[2]}{([1]+[1])} a_{2}^{2} .
\end{aligned}
$$

Using (60) in (66), we have

$$
\begin{aligned}
a_{3}= & \frac{-(A-B)\left(c_{2}-d_{2}\right)}{([1]+[1])\{(1-\beta)+\beta[3]+\gamma[3][2]\} \Psi_{2}} \\
& +\frac{[2]}{([1]+[1])} a_{2}^{2} .
\end{aligned}
$$

Taking the modulus of (67), we have

$$
\begin{aligned}
\left|a_{3}\right| \leq & \frac{|A-B|\left(\left|c_{2}\right|+\left|d_{2}\right|\right)}{([1]+[1])\{(1-\beta)+\beta[3]+\gamma[3][2]\} \Psi_{2}} \\
& +\frac{[2]}{([1]+[1])}\left|a_{2}\right|^{2} .
\end{aligned}
$$


By using the estimates $\left|c_{2}\right| \leq 1-\left|c_{1}\right|^{2},\left|d_{2}\right| \leq 1-\left|d_{1}\right|^{2}$, of Lemma 5 , and $\left|c_{1}\right| \leq 1,\left|d_{1}\right| \leq 1$, in (68), we have

$$
\left|a_{3}\right| \leq \frac{[2]}{([1]+[1])}\left|a_{2}\right|^{2}
$$

and using (56) in (69) we have

$$
\begin{aligned}
& \left|a_{3}\right| \\
& \quad \leq \frac{[2]}{([1]+[1])}\left[\frac{(1-\alpha)(1+q)}{\{(1-\beta)+\beta[2]+\gamma[2][1]\} \Psi_{1}}\right]^{2} .
\end{aligned}
$$

Using (20) and (16) in (70), we have

$$
\begin{aligned}
& \left|a_{3}\right| \\
& \leq \frac{[2]}{([1]+[1])}\left[\frac{(1-\alpha)(1+q)[1] ! \Gamma_{q}(1+\lambda)}{\{(1-\beta)+\beta[2]+\gamma[2][1]\} \Gamma_{q}(2+\lambda)}\right]^{2} .
\end{aligned}
$$

Again using (64) in (69) we have

$$
\begin{aligned}
& \left|a_{3}\right| \\
& \leq \frac{[2]}{([1]+[1])}\left[\frac{2 q(1-\alpha)(1+q)[2] ! \Gamma_{q}(1+\lambda)}{[2]\{(1-\beta)+\beta[3]+\gamma[3][2]\} \Gamma_{q}(3+\lambda)}\right] .
\end{aligned}
$$

From (59) we have

$$
\left|a_{3}-[2] a_{2}^{2}\right| \leq \frac{|(A-B) B|\left|d_{1}\right|^{2}+|A-B|\left|d_{2}\right|}{\{(1-\beta)+\beta[3]+\gamma[3][2]\} \Psi_{2}} .
$$

Using $\left|d_{2}\right| \leq 1-\left|d_{1}\right|^{2},\left|d_{1}\right|$ of Lemma 5 and (20), (16), on (73), we have

$$
\left|a_{3}-[2] a_{2}^{2}\right| \leq \frac{q(1-\alpha)(1+q)[2] ! \Gamma_{q}(1+\lambda)}{\{(1-\beta)+\beta[3]+\gamma[3][2]\} \Gamma_{q}(3+\lambda)} .
$$
lary.

For $\beta=1$, in Theorem 13, we obtain the following corol-

Corollary 14. For $-1<\lambda, 0 \leq \gamma$, and $0 \leq \alpha<1$, if $f \in$ $\mathcal{N}_{\Sigma}^{q(\boldsymbol{\alpha}, \gamma, \lambda)}$, then

$$
\left|a_{2}\right| \leq \min \left\{\begin{array}{l}
\frac{(1-\alpha)(1+q)[1] ! \Gamma_{q}(1+\lambda)}{[2]\{1+\gamma\} \Gamma_{q}(2+\lambda)}, \\
\sqrt{\frac{2 q(1-\alpha)(1+q)[2] ! \Gamma_{q}(1+\lambda)}{[2][3]\{1+\gamma[2]\} \Gamma_{q}(3+\lambda)}},
\end{array}\right.
$$

$\left|a_{3}\right|$

$$
\begin{aligned}
& \leq \min \left\{\begin{array}{l}
\frac{[2]}{([1]+[1])}\left[\frac{(1-\alpha)(1+q)[1] ! \Gamma_{q}(1+\lambda)}{[2]\{1+\gamma[1]\} \Gamma_{q}(2+\lambda)}\right]^{2}, \\
\frac{[2]}{([1]+[1])}\left[\frac{2 q(1-\alpha)(1+q)[2] ! \Gamma_{q}(1+\lambda)}{[2][3]\{1+\gamma[2]\} \Gamma_{q}(3+\lambda)}\right],
\end{array}\right. \\
& \left|a_{3}-[2] a_{2}^{2}\right| \leq \frac{q(1-\alpha)(1+q)[2] ! \Gamma_{q}(1+\lambda)}{[3]\{1+\gamma[2]\} \Gamma_{q}(3+\lambda)} .
\end{aligned}
$$

For $\beta=1$ and $\gamma=0$, in Theorem 13, we obtain the following corollary.

Corollary 15. For $-1<\lambda, 0 \leq \alpha<1$, if $f \in \mathcal{N}_{\Sigma}^{q(\alpha, \gamma, \lambda)}$, then

$$
\left|a_{2}\right| \leq \min \left\{\begin{array}{l}
\frac{(1-\alpha)(1+q)[1] ! \Gamma_{q}(1+\lambda)}{[2] \Gamma_{q}(2+\lambda)}, \\
\sqrt{\frac{2 q(1-\alpha)(1+q)[2] ! \Gamma_{q}(1+\lambda)}{[2][3] \Gamma_{q}(3+\lambda)}},
\end{array}\right.
$$

$\left|a_{3}\right|$

$$
\leq \min \left\{\begin{array}{l}
\frac{[2]}{([1]+[1])}\left[\frac{(1-\alpha)(1+q)[1] ! \Gamma_{q}(1+\lambda)}{[2] \Gamma_{q}(2+\lambda)}\right]^{2}, \\
\frac{[2]}{([1]+[1])}\left[\frac{2 q(1-\alpha)(1+q)[2] ! \Gamma_{q}(1+\lambda)}{[2][3] \Gamma_{q}(3+\lambda)}\right],
\end{array}\right.
$$

$\left|a_{3}-[2] a_{2}^{2}\right| \leq \frac{q(1-\alpha)(1+q)[2] ! \Gamma_{q}(1+\lambda)}{[3] \Gamma_{q}(3+\lambda)}$.

For $\lambda=0, q \rightarrow 1$, in Theorem 13, we obtain the following corollary.

Corollary 16 (see [21]). For $0 \leq \alpha<1,1 \leq \beta$, and $0 \leq \gamma$, if $f \in \mathcal{N}_{\Sigma}^{(\boldsymbol{\alpha}, \boldsymbol{\beta}, \boldsymbol{\gamma})}$, then

$\left|a_{2}\right|$

$$
\leq\left\{\begin{array}{lc}
\sqrt{\frac{2(1-\alpha)}{1+2 \beta+6 \gamma},} & 0 \leq \alpha<1-\frac{(1+\beta+2 \gamma)^{2}}{2(1+2 \beta+6 \gamma)}, \\
\frac{2(1-\alpha)}{1+\beta+2 \gamma}, & 1-\frac{(1+\beta+2 \gamma)^{2}}{2(1+2 \beta+6 \gamma)} \leq \alpha<1,
\end{array}\right.
$$

$$
\begin{aligned}
& \left|a_{3}\right| \leq \frac{2(1-\alpha)}{1+2 \beta+6 \gamma}, \\
& \left|a_{3}-2 a_{2}^{2}\right| \leq \frac{2(1-\alpha)}{1+2 \beta+6 \gamma} .
\end{aligned}
$$

For $\lambda=0, \gamma=0, q \rightarrow 1$, in Theorem 13, we obtain the following corollary.

Corollary 17 (see [30]). For $0 \leq \alpha<1$ and $1 \leq \beta$, if $f \in$ $\mathcal{N}_{\Sigma}^{(\boldsymbol{\alpha}, \boldsymbol{\beta})}$, then

$$
\left|a_{2}\right| \leq \begin{cases}\sqrt{\frac{2(1-\alpha)}{1+2 \beta}}, & 0 \leq \alpha<1-\frac{(1+\beta)^{2}}{2(1+2 \beta)}, \\ \frac{2(1-\alpha)}{1+\beta}, & 1-\frac{(1+\beta)^{2}}{2(1+2 \beta)} \leq \alpha<1,\end{cases}
$$

$$
\begin{array}{r}
\left|a_{3}\right| \leq \frac{2(1-\alpha)}{1+2 \beta}, \\
\left|a_{3}-2 a_{2}^{2}\right| \leq \frac{2(1-\alpha)}{1+2 \beta} .
\end{array}
$$

For $\lambda=0, \beta=1, \gamma=0, q \rightarrow 1$, in Theorem 13, we obtain the following [21]. 
Corollary 18 (see [21]). For $0 \leq \alpha<1$, if $f \in \mathscr{H}_{\Sigma}^{(\alpha)}$, then

$$
\begin{aligned}
\left|a_{2}\right| & \leq \begin{cases}\sqrt{\frac{2(1-\alpha)}{3},} & 0 \leq \alpha<\frac{1}{3}, \\
(1-\alpha), & \frac{1}{3} \leq \alpha<1 .\end{cases} \\
\left|a_{3}\right| & \leq \frac{2(1-\alpha)}{3}, \\
\left|a_{3}-2 a_{2}^{2}\right| & \leq \frac{2(1-\alpha)}{3} .
\end{aligned}
$$

For $\lambda=0, q \rightarrow 1, \beta=1$, in Theorem 13, we obtain the following corollary.

Corollary 19 (see [25]). For $0 \leq \alpha<1,1 \leq \beta$, and $0 \leq \gamma$, if $f \in \mathscr{B}_{\Sigma}^{(\boldsymbol{\alpha}, \gamma)}$, then

$$
\begin{aligned}
& \left|a_{2}\right| \leq \begin{cases}\sqrt{\frac{2(1-\alpha)}{3(1+2 \gamma)}}, & 0 \leq \alpha<\frac{1+2 \gamma-2 \gamma^{2}}{3(1+2 \gamma)}, \\
\frac{2(1-\alpha)}{1+2 \gamma}, & \frac{1+2 \gamma-2 \gamma^{2}}{3(1+2 \gamma)}, \leq \alpha<1 .\end{cases} \\
& \left|a_{3}\right| \leq \frac{2(1-\alpha)}{3(1+2 \gamma)} .
\end{aligned}
$$

\section{Disclosure}

The present address of Zahid Shareef is Division of Engineering, Higher Colleges of Technology, P.O. Box 4114, Fujairah, UAE.

\section{Conflicts of Interest}

The authors declare that they have no conflicts of interest.

\section{Acknowledgments}

This work is supported by MOHE Grant FRGS/1/2016/ STG06/UKM/01/1.

\section{References}

[1] P. L. Duren, Univalent Functions, Grundehren der Math. Wiss, Springer, New York, NY, USA, 1983.

[2] H. M. Srivastava, A. K. Mishra, and P. Gochhayat, "Certain subclasses of analytic and bi-univalent functions," Applied Mathematics Letters. An International Journal of Rapid Publication, vol. 23, no. 10, pp. 1188-1192, 2010.

[3] D. A. Brannan and J. Clunie, "Aspects of contemporary complex analysis," in Proceedings of the NATO Advanced study Institute Held at University of Durham, Academic Press, New York, NY, USA, 1979.

[4] E. Netanyahu, "The minimal distance of the image boundary from the origin and the second coefficient of a univalent function in $-\mathrm{z}-<1$," Archive for Rational Mechanics and Analysis, vol. 32, no. 2, pp. 100-112, 1969.

[5] D. A. Brannan and T. S. Taha, "On some classes of bi-univalent functions," Studia Universitatis Babes-Bolyai Mathematica, vol. 31, no. 2, pp. 70-77, 1986.
[6] S. Altinkaya and S. Yalcin, "Initial coefficient bounds for a general class of biunivalent functions," International Journal of Analysis, vol. 2014, Article ID 867871, 4 pages, 2014.

[7] B. S. Keerthi and B. Raja, "Coefficient inequality for certain new subclasses analytic bi-univalent functions," Theory. Math. Appl, vol. 3, no. 1, pp. 1-10, 2013.

[8] M. Lewin, "On a coefficient problem for bi-univalent functions," Proceedings of the American Mathematical Society, vol. 18, pp. 63-68, 1967.

[9] X.-F. Li and A.-P. Wang, "Two new subclasses of bi-univalent functions," International Mathematical Forum, vol. 7, pp. 14951504, 2012.

[10] N. Magesh and J. Yamini, "Coefficient bounds for certain subclasses of bi-univalent functions," International Mathematical Forum. Journal for Theory and Applications, vol. 8, no. 25-28, pp. 1337-1344, 2013.

[11] S. Porwal and M. Darus, "On a new subclass of bi-univalent functions," Journal of the Egyptian Mathematical Society, vol. 21, no. 3, pp. 190-193, 2013.

[12] G. Faber, "Uber polynomische Entwickelungen," Mathematische Annalen, vol. 57, no. 3, pp. 1569-1573, 1903.

[13] S. Gong, "The Bieberbach conjecture,translated from the 1989 Chinese original and revised by the author," in AMS/IP Studies in Advanced Mathematics, vol. 12, American Mathematical Society, Providence, RI, USA, 1999.

[14] M. Schiffer, "Surun probleme d'extr emum de la representation conforme," Bull. Soc. Math. France, vol. 66, pp. 48-55, 1938.

[15] H. Airault, "Remarks on Faber polynomials," International Mathematical Forum, vol. 3, no. 9, pp. 449-456, 2008.

[16] H. Airault and A. Bouali, "Differential calculus on the Faber polynomials," Bulletin des Sciences Mathématiques, vol. 130, no. 3, pp. 179-222, 2006.

[17] S. Bulut, "Faber polynomial coefficient estimates for a comprehensive subclass of analytic bi-univalent functions," Comptes Rendus Mathematique, vol. 352, no. 6, pp. 479-484, 2014.

[18] S. G. Hamidi and J. M. Jahangiri, "Faber polynomial coefficient estimates for analytic bi-close-to-convex functions," Comptes Rendus Mathematique, vol. 352, no. 1, pp. 17-20, 2014.

[19] S. G. Hamidi, S. Abd Halim, and J. M. Jahangiri, "Faber polynomial coefficient estimates for meromorphic Bi-starlike functions," International Journal of Mathematics and Mathematical Sciences, vol. 2013, Article ID 498159, 2013.

[20] S. G. Hamidi and J. M. Jahangiri, "Faber polynomial coefficients of bi-subordinate functions," Comptes Rendus Mathematique, vol. 354, no. 4, pp. 365-370, 2016.

[21] S. Bulut, "Faber polynomial coefficient estimates for a subclass of analytic bi-univalent functions," Filomat, vol. 30, no. 6, pp. 1567-1575, 2016.

[22] S. Ruscheweyh, "New criteria for univalent functions," Proceedings of the American Mathematical Society, vol. 49, pp. 109-115, 1975.

[23] S. Kanas and D. Răducanu, "Some class of analytic functions related to conic domains," Mathematica Slovaca, vol. 64, no. 5, pp. 1183-1196, 2014.

[24] H. Aldweby and M. Darus, "Some subordination results on $q$-analogue of Ruscheweyh differential operator," Abstract and Applied Analysis, vol. 2014, Article ID 958563, 6 pages, 2014.

[25] H. M. Srivastava, S. S. Eker, and R. M. Ali, "Coefficient bounds for a certain class of analytic and bi-univalent functions," Filomat, vol. 29, no. 8, pp. 1839-1845, 2015. 
[26] B. A. Frasin and M. K. Aouf, "New subclasses of bi-univalent functions," Applied Mathematics Letters. An International Journal of Rapid Publication, vol. 24, no. 9, pp. 1569-1573, 2011.

[27] F. R. Keogh and E. P. Merkes, "A coefficient inequality for certain classes of analytic functions," Proceedings of the American Mathematical Society, vol. 20, pp. 8-12, 1969.

[28] H. Airault, "Symmetric sums associated to the factorizations of Grunsky coefficients," in Proceedings of the Groups and Symmetries, Montreal, Canada, April 2007.

[29] H. Airault and J. Ren, "An algebra of differential operators and generating functions on the set of univalent functions," Bulletin des Sciences Mathématiques, vol. 126, no. 5, pp. 343-367, 2002.

[30] J. M. Jahangiri and S. G. Hamidi, "Coefficient estimates for certain classes of bi-univalent functions," International Journal of Mathematics and Mathematical Sciences, vol. 2013, Article ID 190560, 2013. 


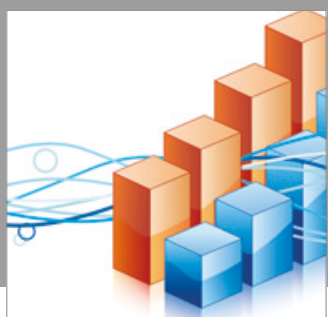

Advances in

Operations Research

vatersals

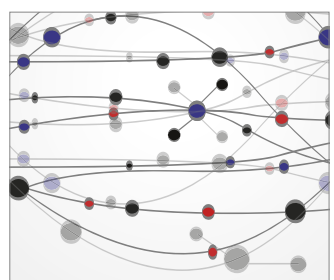

\section{The Scientific} World Journal
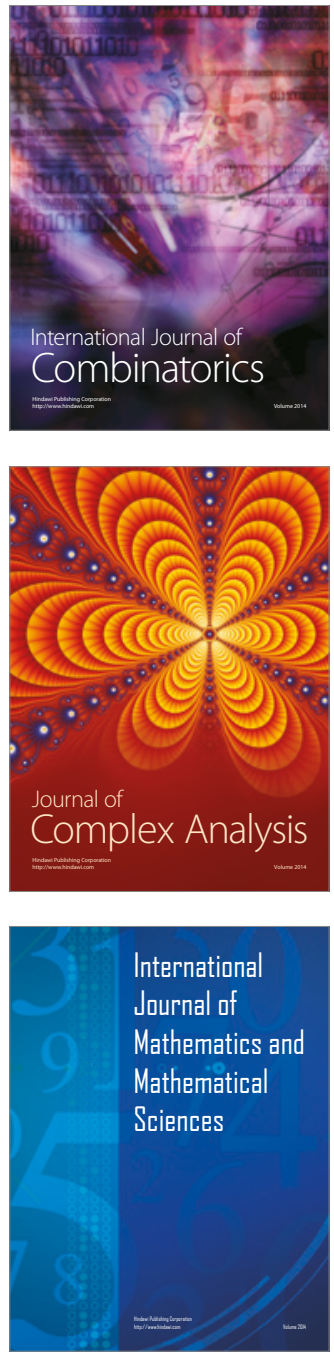
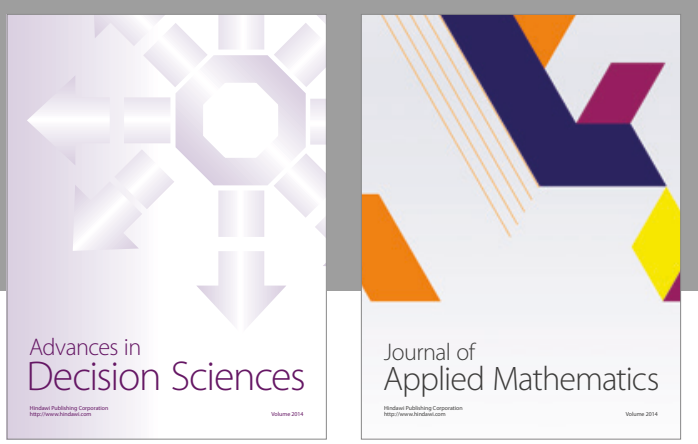

Algebra

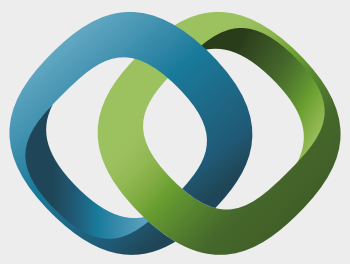

\section{Hindawi}

Submit your manuscripts at

https://www.hindawi.com
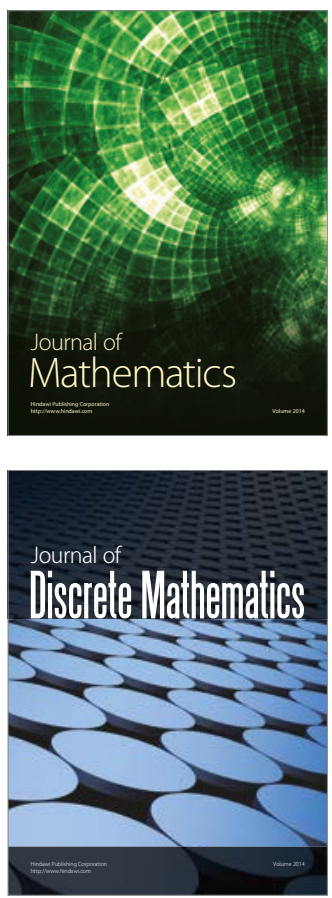

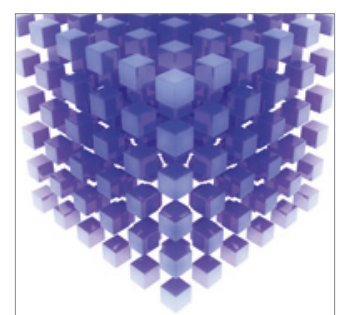

Mathematical Problems in Engineering
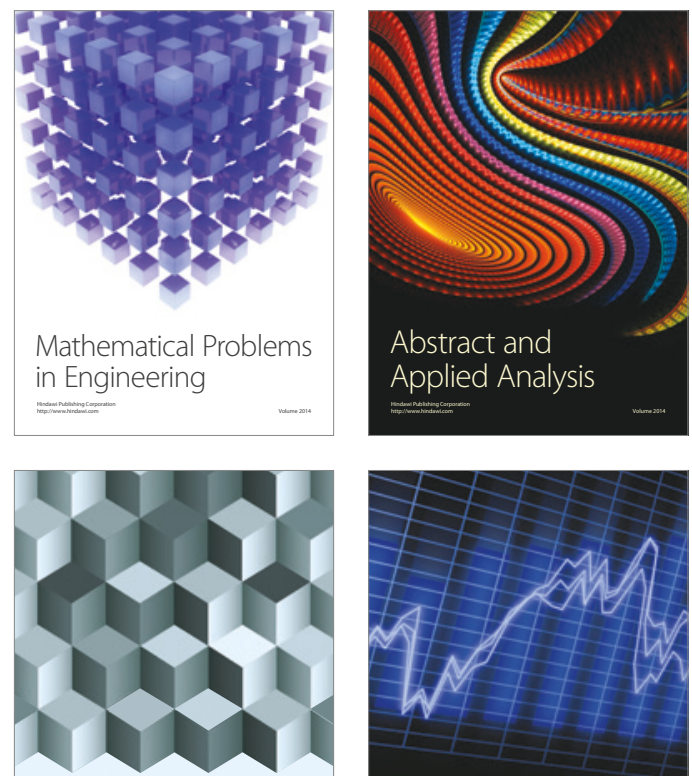

Journal of

Function Spaces

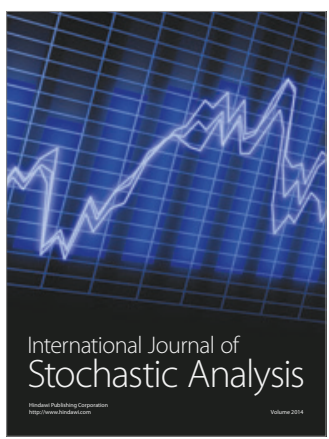

Probability and Statistics
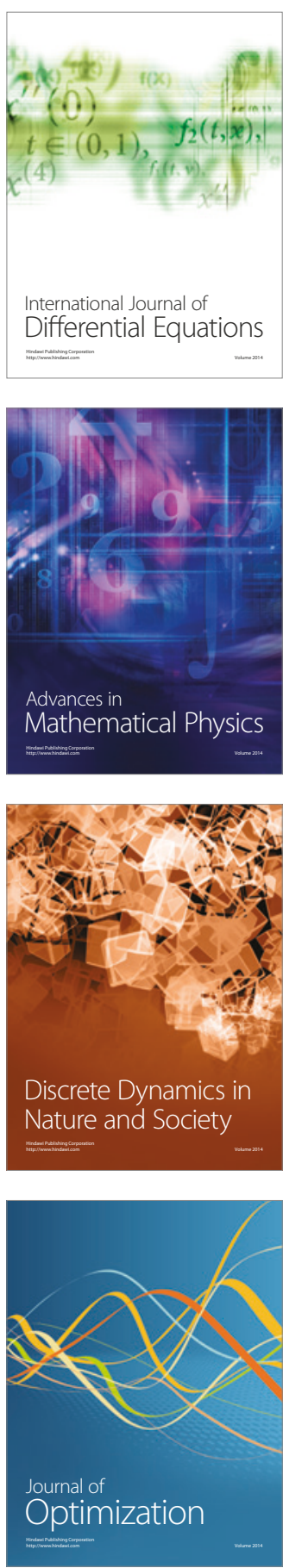